\title{
Belphégor
}

\section{Mathieu de Wasseige, Séries télé US : l'idéologie prime}

time

\section{Stéphane Benassi}

\section{(2) OpenEdition}

1 Journals

\section{Édition électronique}

URL : http://journals.openedition.org/belphegor/537

DOI : 10.4000/belphegor.537

ISSN : 1499-7185

Éditeur

LPCM

\section{Référence électronique}

Stéphane Benassi, « Mathieu de Wasseige, Séries télé US : l'idéologie prime time », Belphégor [En ligne], 13-1 | 2015, mis en ligne le 09 mai 2015, consulté le 22 septembre 2020. URL : http:// journals.openedition.org/belphegor/537 ; DOI : https://doi.org/10.4000/belphegor.537

Ce document a été généré automatiquement le 22 septembre 2020.

\section{(c) (i) (9)}

Belphégor est mis à disposition selon les termes de la Licence Creative Commons Attribution - Pas d'Utilisation Commerciale - Pas de Modification 4.0 International. 


\section{Mathieu de Wasseige, Séries télé US : l'idéologie prime time}

\section{Stéphane Benassi}

\section{RÉFÉRENCE}

Mathieu De Wasseige (avec la collaboration de Barbara Dupont), Séries télé US : l'idéologie prime time, Louvain-la-Neuve, Academia/L'Harmattan S.A., coll. « IHECS [dot] COM », 2015.ISBN : 978-2-8061-0176-1 
1 Se situant au cœur des cultural studies anglo-saxonnes, la question des « représentations » est sans doute celle qui est la plus répandue dans la littérature académique consacrée aux fictions télévisuelles. L'ouvrage de Mathieu de Wasseige, américaniste chargé de cours à l'Institut des Hautes Études des Communications Sociales de Bruxelles, n'échappe pas à cette tendance qui, au fil des années, a fini par focaliser l'attention de la recherche sur cette seule question en délaissant bien souvent certains autres aspects, parfois plus essentiels, de l'étude des fictions plurielles de la télévision. En effet, si les cultural studies ont montré qu'elles pouvaient être le lieu au sein duquel serait susceptible de se développer une approche critique de ces fictions, elles

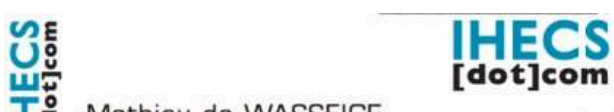

플 Mathieu de WASSEIGE [dot]com SÉRIES TÉLÉ US :

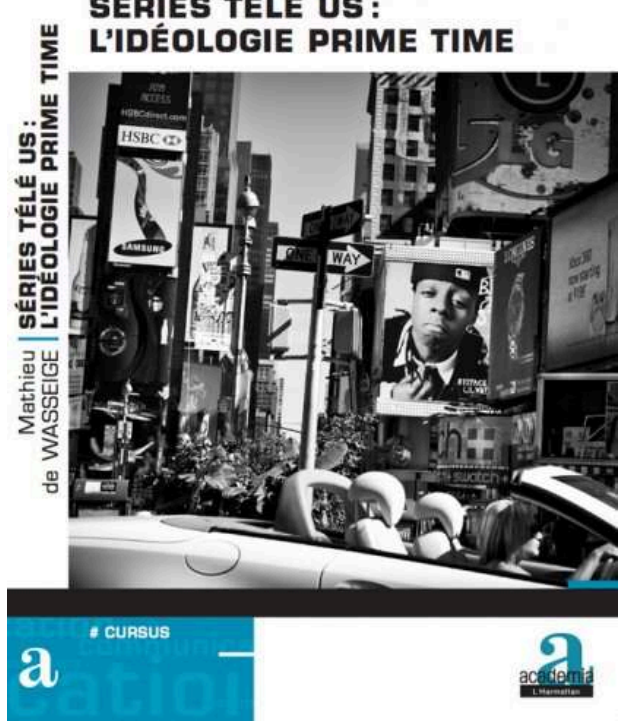
ont aussi parfois montré certaines de leurs limites en négligeant par exemple les dimensions narratives, temporelles ou économiques, pourtant centrales dans la relation que ces productions culturelles établissent avec leurs publics.

Conscient de cela, l'auteur envisage la question de la représentation du monde sous son aspect idéologique en précisant que celui-ci ne serait qu'un facteur, parmi de nombreux autres (qu'il n'identifie pas), susceptible de jouer un rôle dans le succès d'une série télévisée. Le propos de son essai consiste donc à montrer qu'il pourrait exister un lien entre le type d'idéologie véhiculé par une série et la popularité dont elle bénéficie auprès du public. Partant du principe que la télévision, au sens large, est le vecteur le plus important de représentation du monde et une source d'inspiration fondamentale pour notre perception de ce dernier, il se fixe un double objectif : d'une part montrer que même les séries les plus populaires des networks américains, toutes programmées en Europe, ne sont pas des fictions consensuelles qui n'abordent que des sujets fédérateurs et représentent un monde très éloigné de certaines réalités sociales; d'autre part nourrir la recherche sur «l'éducation aux médias » en développant le concept d'« idéologie prime time » sur la base du balancement idéologique qu'il entend mettre en évidence à travers ses analyses. Après deux courts chapitres respectivement consacrés aux cultural studies et aux études télévisuelles destinés à poser dans les grandes lignes le cadre théorique de sa réflexion (de façon parfois un peu trop expéditive), de Wasseige aborde dans les trois chapitres suivants les principales questions de société traités dans certaines séries récentes diffusées sur les grands networks américains (Grey's Anatomy, Desperate Housewives, 24 heures chrono, À la Maison Blanche, Cold Case, Les experts ou Glee) en les regroupant au sein de trois grandes thématiques: "sécurité nationale et terrorisme", "genre et sexualité ", "marges sociales ».

3 Si l'auteur choisit de focaliser sa réflexion sur les questions de représentation et d'idéologie, et sur les structures sociales dans lesquelles elles prennent place, c'est, 
explique-t-il dans le premier chapitre, parce que ces questions sont centrales dans les études télévisuelles et dans les cultural studies, et en particulier dans l'analyse des séries télévisées qui, selon lui, seraient l'exemple même de l'affaiblissement de la distinction entre culture élitiste et culture de masse, aussi bien dans leur dimension esthétique que dans leur dimension sociale et politique. Considérant les séries télévisées comme des productions représentatives de la culture populaire en tant que moyen d'émancipation et non de domination comme le postulaient les théoriciens de l'École de Francfort, de Wasseige explique en outre qu'il s'intéressera plus spécifiquement à la façon dont elles construisent des représentations particulières de la société, donc des idéologies au sens où l'entendent Louis Althusser ou Antonio Gramsci. S'il fonde sa réflexion sur les travaux de John Fiske, Stuart Hall, Frederic Jameson ou Richard Dyer et les théories associées aux communautés interprétatives, l'auteur refuse toute vision angélique de la culture populaire en rappelant fort justement que les industries culturelles sont avant tout mues par des intérêts économiques qui les conduisent nécessairement à manipuler leurs publics, ne fût-ce que par leur contrôle sur la production, afin de s'assurer autant que possible un profit financier. En somme, la culture populaire serait une confrontation entre les industries de la culture et le public, caractérisée par un combat permanent entre des forces de fermeture et d'ouverture idéologiques.

Cette approche économique de la télévision en tant qu'industrie culturelle constitue le cœur du deuxième chapitre dans lequel l'auteur propose un rapide ancrage des séries au sein des études télévisuelles. Cela lui permet en outre de légitimer le choix de son corpus, en précisant que si celui-ci porte exclusivement sur certaines séries (généralement désignées par l'appellation drama bien que le terme ne soit pas utilisé) des grandes chaînes généralistes américaines, c'est parce qu'elles s'adressent à un large public tout en étant soumises aux exigences d'annonceurs majoritairement conservateurs qui rejettent souvent les récits trop engagés et les images trop choquantes par peur de déranger le consommateur potentiel, ainsi qu'aux limitations imposée par la FCC, l'agence de contrôle de la télévision. Cela lui permet également de définir l'objectif principal de son travail qui sera, dit-il, de déchiffrer les stratégies présentes dans toutes les séries télévisées des networks américains qui constituent ce qu'il nomme «l'idéologie prime time » et qu'il définit comme étant le résultat contrasté du compromis entre les velléités souvent opposées des créateur et des producteurs de ces séries. Toutefois, de Wasseige justifie également son choix en ce référant au concept discutable de Quality TV popularisé par Robert J. Thompson, et met en place une méthodologie d'analyse selon nous hasardeuse qui le conduit non seulement à tirer des conclusions générales en se basant sur l'étude d'un nombre limité d'exemples, mais aussi à fonder ses analyses sur un nombre réduit épisodes isolés plutôt que sur chaque œuvre dans son ensemble, chaque série considérée en tant que tout signifiant, et sans tenir compte des différentes formes syntaxiques et narratives des fictions plurielles da la télévision.

5 En se focalisant sur les thématiques de la sécurité nationale et du terrorisme, et en développant une analyse comparée de 24 heures chrono et À la Maison Blanche, le troisième chapitre s'attache à mettre en évidence l'importance de thèmes contemporains dans la fiction télévisuelle comme moyen d'aviser le public quant à des thématiques sociétales particulières, mais aussi comme marqueurs du biais idéologique inévitable et souvent délibéré des séries. En dépit d'un certain nombre d'imprécisions dans le maniement des concepts de la narratologie, l'auteur y montre en l'occurrence que malgré une dynamique narrative, une orientation politique et des cibles distinctes 
liés aux chaînes qui les diffusent, ces deux séries proposent une idéologie explicite. Le fait que ces programmes s'inscrivent dans l'actualité démontre pour l'auteur que les thématiques « délicates » peuvent trouver leur place dans la fiction américaine grand public, et ne sont ni voilées, ni autocensurées. Selon lui, la présence de ces séries en prime time est cruciale, car elles éveillent l'intérêt et l'attention de leurs spectateurs en les confrontant aux problèmes moraux qu'elles abordent, et constituent donc de bonnes amorces au débat sur les enjeux idéologiques de notre société, tant au niveau politique que, plus largement, au niveau social.

6 Les questions du genre et de la sexualité, elles aussi centrales dans les cultural studies, constituent les thématiques principales du quatrième chapitre. Après avoir observé la façon dont 24 heures chrono et À la Maison Blanche développent une représentation différente des femmes, conservatrice pour l'une, plus progressiste pour l'autre, en accord avec l'orientation politique des chaînes qui produisent ces séries, de Wasseige s'attarde plus longuement sur l'exemple de Desperate Housewives. Considérant cette série (ce feuilleton, devrait-on plutôt dire) comme un exemple parfait de programme fragmenté destiné à un public aussi large que possible en usant de l'« excès sémiotique ", il tente de montrer que sa dimension "populaire » est avant tout liée au mélange des genres (diégétiques) qu'elle propose et au fait qu'elle navigue sur une idéologie hésitante, avec des personnages volontairement ambivalents. Ainsi met-il en évidence le fait que si son orientation idéologique générale est "résolument conservatrice", Desperate Housewives incorpore néanmoins certains éléments de concession dans de nombreux épisodes. Si, en se concentrant sur les femmes au foyer, la série semble marquer un recul face aux représentations plus positives des femmes observables chez nombre de ses consœurs, ses protagonistes féminines seraient toutefois des exemples typiques de l'ère post-féministe. L'auteur propose ensuite, en dépit de l'absence de tout point commun entre ces deux œuvres, une analyse comparée de Desperate Housewives et Grey's Anatomy au terme de laquelle il conclut qu'avec leurs approches distinctes du statut contemporain de la femme, elles offrent différents éclairages conceptuels pour aborder le post-féminisme, et se rejoignent dans ce que Angela McRobbie appelle le double-enchevêtrement, c'est-à-dire la coexistence dans la culture populaire de valeurs néoconservatrices liées au genre, à la sexualité ou à la vie de famille, avec des processus de progressisme vis-à-vis du choix de la diversité des relations domestiques, sexuelles ou familiales. Enfin, c'est Barbara Dupont qui prend la plume pour montrer, à travers l'exemple de la série pour adolescents Glee, que la fiction télévisuelle, en tant que catégorie de la culture populaire, contribue pleinement à mettre au centre du débat public des sujets qui ne sont que difficilement ou caricaturalement traités ailleurs, en particulier aux États-Unis.

7 Toutefois, si de nombreux thèmes de société jusqu'alors peu abordés sur les networks ont progressivement été intégrés aux fictions de prime time ces dernières décennies, cette évolution délaisse certaines catégories, telles que les "mages sociales » qui, encore aujourd'hui, sont largement négligées dans les séries télévisées américaines. Pour aborder cette dernière thématique, le chapitre cinq se focalise sur les séries policières que l'auteur considère à la suite de Nathalie Perreur comme des tentatives fictionnelles cherchant à englober de manière réflexive et critique la société contemporaine, en incorporant à leurs récits tout ce qui fait matière à un débat sociale. Après avoir constaté la présence diversifiée de ces mages sociales (minorités ethniques, homosexualité, addiction aux drogues, à l'alcool ou aux médicaments, etc.) dans certains épisodes des séries précédemment prises en exemple, de Wasseige s'attache à 
montrer comment celles-ci sont abordées dans Les Experts et Cold Case, deux des séries policières les plus populaires de ces dernières années. La première, constate-t-il, développe deux positions idéologiques opposées, qui peuvent être perçues comme un cas de balancement idéologique presque parfait. En effet, d'un côté la série représente un monde beaucoup plus dangereux qu'il ne l'est réellement, promeut l'infaillibilité de la recherche médico-légale et d'un système punitif ce qui trahit une inclinaison conservatrice, et d'un autre côté, elle surreprésente les meurtriers caucasiens et montre de nombreuses minorités souvent coopératives et rendues légitimes par le récit et les personnages principaux, ce qui relève davantage d'un inclinaison progressiste. Selon l'auteur, Cold Case s'inscrit en contraste avec Les Experts dans la mesure où elle serait pour sa part un exemple rare de programme extrêmement progressiste qui ne propose que peu de concessions aux conservateurs, et pas de balancement idéologique significatif. La série se démarque par sa représentation de nombreuses minorités qu'elle défend systématiquement et décrit de façon particulièrement positive, mais également par une représentation nuancée de la police. L'analyse de ces derniers exemples permet à de Wasseige d'affiner son concept d'« idéologie prime time » qu'il définit alors comme la combinaison de postures idéologiques, parfois plurielles mais rarement faibles, et de (contre-) balancement idéologique.

8 En dépit de quelques interprétations à l'emporte-pièce dues à un ancrage théorique trop superficiel sur certains aspects, et de références qui ne sont pas toujours précises ni intégrées à la bibliographie, cet ouvrage à toutefois le mérite de proposer et de développer le concept d'" idéologie prime time ", qui pourra sans doute s'avérer utile pour l'analyse des fictions plurielles de la télévision. Cette idéologie, véhiculée par les networks américains dans leurs séries télévisées, n'est pas une idéologie molle, tiède ou consensuelle. C'est au contraire une idéologie claire, faisant parfois même preuve d'un engagement politique marqué, mais toujours contrebalancé dans une certaine mesure par des personnages, des histoires ou des pratiques contraires à cette ligne directrice. Elle s'explique par la volonté des networks de produire des fictions susceptibles de plaire au plus grand nombre en assumant généralement une ligne idéologique évidente via l'histoire ou la représentation du monde qu'elle offre, et en proposant simultanément des éléments qui ne paraissent pas (directement) contradictoires. L'auteur constate d'ailleurs que parmi les séries analysées, ce sont systématiquement celles qui sont les plus marquées idéologiquement qui sont les moins populaires, montrant ainsi la corrélation entre la grandeur du succès rencontré par une série et l'importance de son degré de balancement idéologique. 